\title{
NUMERICAL STUDY OF MESOSCALE CELLULAR CONVECTION
}

\author{
WU-RON HSU
}

Department of Atmospheric Sciences, National Taiwan University, Taipei, Taiwan, R.O.C.

and

WEN-YIH SUN

Department of Earth and Atmospheric Sciences, Purdue University, West Lafayette, IN 47907, U.S.A.

(Received in final form 15 May, 1991)

\begin{abstract}
Mesoscale cellular convections over the East China Sea during cold air outbreaks are simulated with a high-resolution numerical model. The model incorporates important physical processes involved in shallow convection, such as the exchange of heat and moisture between water and air; condensation; evaporation; and vertical turbulent transfer of heat, moisture, and momentum.

The results show that open cells develop with aspect ratios as large as 14 . The structure of the convection is examined in detail. The organized mesoscale circulation is responsible for breaking up the initial stratus cloud deck and enhancing turbulence in the upward-moving area (especially inside cumulus clouds). However, it is found that the heat flux contributed by MCC's themselves is much smaller than the eddy heat flux.
\end{abstract}

\section{Introduction}

Mesoscale cellular convection (MCC) occurs primarily in a marine boundary layer as an extensive, organized array of hexagonal convection cells. These cells have diameters of $10-100 \mathrm{~km}$, and can occur as either open or closed circulation systems (Agee et al., 1973). Open cells have downward motion and clear skies at cell center with upward motion and clouds in the periphery of the hexagon, while closed cells have the opposite circulation. Open cell patterns are typically found behind cold fronts over warm water, such as those shown in Figure 1. Although MCC can often be seen in conventional satellite cloud pictures, detailed structure is difficult to observe, due to the small spatial scale of MCC.

Thermal convection has been studied by many scientists following Benard's (1901) experiment and Rayleigh's (1916) theoretical work. Despite the geometrical similarity between cellular cloud patterns and laboratory observations of cellular convection, they are quite different in several respects:

(1) Aspect ratio (defined as horizontal scale divided by vertical scale of convection) of MCC is one order larger than Benard's convection.

(2) Although the convective boundary layer (CBL) is capped by a very stable inversion layer, the convection can penetrate through the inversion (Sun, 1976).

(3) Because potential temperature above the CBL is higher than that within the CBL, entrainment can serve as a heat source, rather than heat being extracted from the top of the fluid as in Rayleigh's model. Fiedler (1984) showed that 


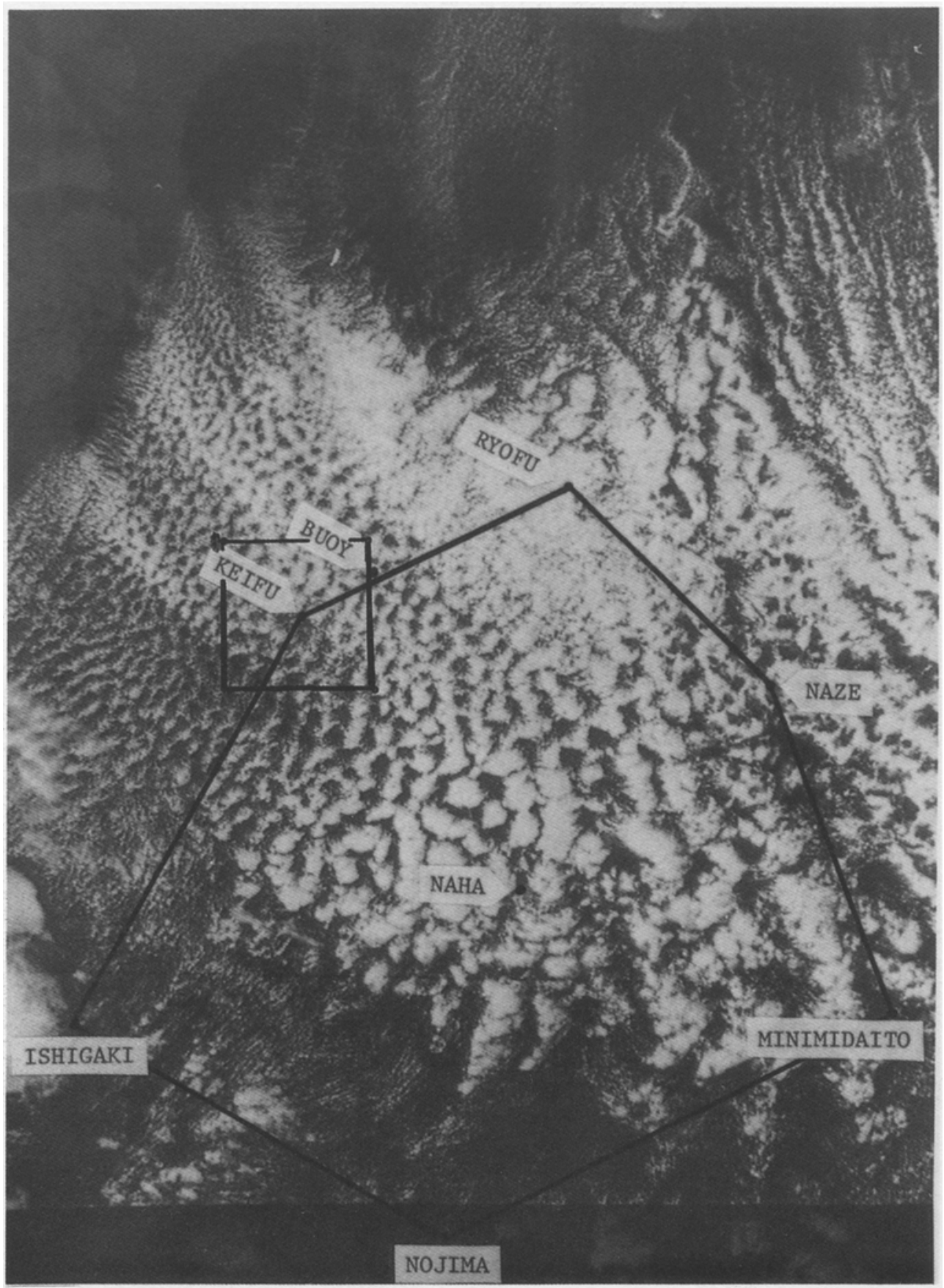

Fig. 1. DMSP satellite visible imagery over the East China Sea at 00Z February 16, 1975. Small square box $(140 \mathrm{~km}$ by $140 \mathrm{~km})$ depicts the 3-D model domain. Hexagon shows the AMTEX observational network. 
instability induced by entrainment is responsible for the large atmospheric convection.

(4) Latent heating and radiation are likely to play an important role in atmospheric convection.

(5) Atmospheric diffusion is by turbulent eddies instead of molecular motion. Eddy coefficients are functions of height. Also, the eddy diffusion process is not isotropic (Priestley, 1962).

(6) Synoptic (or large-scale) weather systems can also influence the MCC. For the CBL over the East China Sea, a steady state is achieved by the balance of energy between heat supplied from the water and large-scale cold air advection (Ninomiya, 1975). Hence, the convection, such as in the case shown in Figure 1, can persist for several days.

Krishnamurti $(1975 a, b)$ investigated the stability of a horizontal layer of fluid in which an adverse temperature gradient and uniform large-scale sinking motion were prescribed. Although latent heating was considered in her mathematical model, entrainment and variation of eddy diffusivity with height were ignored. Kuo and Sun (1976), Asai and Nakamura (1978), and Moeng and Arakawa (1980) simulated atmospheric thermal convection with two-dimensional numerical models. Deardorff (1974a, b and 1980), and Sommeria and LeMone (1978) applied three-dimensional models to simulate the development of the CBL. But the domains of these models were too small to simulate MCC. Sykes et al. (1988) simulated large convective rolls (cloud streets) in the CBL with a three-dimensional model.

In this paper, we report a numerical simulation of "open" MCC's over warm water in the winter with a three-dimensional model. The structure of MCC and its role in transporting heat and moisture from the water's surface are examined in detail. The work provides evidence of a possible mechanism through which the cells appeared open.

All major physical processes related to MCC (such as entrainment, fluxes from water surface, latent heating, etc.) are considered in the model except radiation and large-scale cold air advection, which were eliminated for the sake of simplicity. Since heating from the water's surface provides an unstable condition for convection in this study, radiation may not be important. This is supported by Ninomiya (1975).

\section{Numerical Model}

2.1. The governing EQuations

A set of primitive equations in a normalized pressure coordinate $\sigma$ is used, where $\sigma$ is defined as: 


$$
\sigma=\left(p-p_{t}\right) /\left(p_{s}-p_{t}\right)
$$

where $p$ is the pressure, $p_{t}$ is the constant pressure at the top of the domain, and $p_{s}$ is the surface pressure. This set of equations has been presented in detail by Sun and Hsu (1988). The horizontal momentum equations are:

$$
\frac{\partial u}{\partial t}=\operatorname{Adv}(u)+f v-\frac{1}{p_{*}}\left[\frac{\partial\left(\phi p_{*}\right)}{\partial x}-\frac{\partial(\phi \sigma)}{\partial \sigma} \frac{\partial p_{*}}{\partial x}\right]+\operatorname{Diff}(u)
$$

and

$$
\frac{\partial v}{\partial t}=\operatorname{Adv}(v)-f u-\frac{1}{p_{*}}\left[\frac{\partial\left(\phi p_{*}\right)}{\partial y}-\frac{\partial(\phi \sigma)}{\partial \sigma} \frac{\partial p_{*}}{\partial y}\right]+\operatorname{Diff}(v) .
$$

$\operatorname{Adv}()$, the operator for advection, is defined as:

$$
\operatorname{Adv}()=-u \frac{\partial()}{\partial x}-v \frac{\partial()}{\partial y}-\dot{\sigma} \frac{\partial()}{\partial \sigma},
$$

where $u, v$, and $\dot{\sigma}$ are velocity components along the $x, y$, and $\sigma$ coordinates, respectively, and $f$ is the Coriolis parameter. The third term, in brackets on the right-hand side of Equations (2) and (3) is the pressure gradient force in which $\phi$ is geopotential height ( $\phi=g z$, where $g$ is the gravitational acceleration). $p_{*}$ is defined as:

$$
p_{*}=p_{s}-p_{t}
$$

The last term in the momentum equations is the diffusion term derived from mixing-length theory. (The diffusion operator, $\operatorname{Diff}($ ), will be defined later.)

For the thermodynamic variable, equivalent potential temperature, $\theta_{e}$, was employed,

$$
\theta_{e}=\theta+\left(l_{v} / c_{p}\right)(\theta / T) q
$$

where $\theta$ is potential temperature, $l_{v}$ is the latent heat of vaporization, $c_{p}$ is the specific heat at constant pressure, $T$ is temperature, and $q$ is specific humidity. For shallow convection without precipitation, $\theta_{e}$ is a semi-conservative quantity, since $\left(l_{y} / c_{p}\right)(\theta / T)$ is essentially a constant.

$$
\frac{\partial \theta_{e}}{\partial t}=\operatorname{Adv}\left(\theta_{e}\right)+\operatorname{Diff}\left(\theta_{e}\right) .
$$

The moisture equation is as follows:

$$
\frac{\partial q_{w}}{\partial t}=\operatorname{Adv}\left(q_{w}\right)+\operatorname{Diff}\left(q_{w}\right)
$$

where the total specific humidity, $q_{w}=q+q_{l}\left(q_{l}\right.$ is specific water content $)$, is also a semi-conservative quantity in the absence of precipitation. 
The diffusion operator, $\operatorname{Diff}()$, is defined as:

$$
\operatorname{Diff}(\psi)=\frac{\partial}{\partial z}\left(K \frac{\partial \psi}{\partial z}\right)
$$

where

$$
K= \begin{cases}K_{m}, & \text { for } \psi=u, \text { or } v \\ K_{h}, & \text { for } \psi=\theta_{e}, \text { or } q_{w} .\end{cases}
$$

In the above expression, $K_{m}$ and $K_{h}$ are the eddy coefficients for momentum and heat, respectively.

The hydrostatic approximation is adopted in the model because of the large aspect ratios (10-30) for MCC (Agee et al., 1973; Miura, 1985).

$$
\partial \phi / \partial(\ln p)=-R_{d} T\left(1+0.61 q-q_{l}\right),
$$

where $R_{d}$ is the gas constant for dry air. The values of $\theta, q, q_{l}$, and $T$ are calculated as functions of $\theta_{e}, q_{w}$, and $p$ by using Deardorff's (1976) formula.

The equation of conservation of mass is given by:

$$
\frac{\partial p_{*}}{\partial t}=-\int_{0}^{1} \nabla_{\sigma} \cdot\left(p_{*} \mathbf{V}\right) \mathrm{d} \sigma,
$$

where

$$
\nabla_{\sigma} \cdot\left(p_{*} \mathbf{V}\right)=\frac{\partial\left(u p_{*}\right)}{\partial x}+\frac{\partial\left(v p_{*}\right)}{\partial y}
$$

is the divergence of horizontal mass transport. The vertical motion, $\dot{\sigma}$, is solved diagnostically by:

$$
\dot{\sigma}=-\frac{1}{p_{*}} \int_{0}^{r} \nabla_{\sigma} \cdot\left(p_{*} \mathbf{V}\right) \mathrm{d} \sigma^{*}+\frac{\sigma}{p_{*}} \int_{0}^{1} \nabla_{\sigma} \cdot\left(p_{*} \mathbf{V}\right) \mathrm{d} \sigma^{*},
$$

where $\mathrm{d} \sigma^{*}$ is the dummy differential quantity for $\sigma$.

In summary, there are five prognostic equations for $u, v, \theta_{e}, q_{w}$, and $p_{*}$ and two diagnostic relations for $\phi$ and $\dot{\sigma}$. The seven equations corresponding to these seven variables represent a closed set of equations when the eddy coefficients $K_{m}$ and $K_{h}$ are suitably parameterized. This is accomplished by a modified level-2.5 turbulence closure scheme (Sun and Hsu, 1988; and Sun and Wu, 1987).

\subsection{DOMAIN AND GRID SYSTEM}

The domain of the model is a $140 \mathrm{~km}$ by $140 \mathrm{~km}$ by approximately $3 \mathrm{~km}$ box centered at station Keifu $\left(28.11^{\circ} \mathrm{N}, 124.56^{\circ} \mathrm{E}\right)$ over the East China Sea. The pressure at the top is $700 \mathrm{mb}$. In Figure 1, the small box enclosed by the heavy solid lines shows the horizontal dimensions and location of the domain. The 


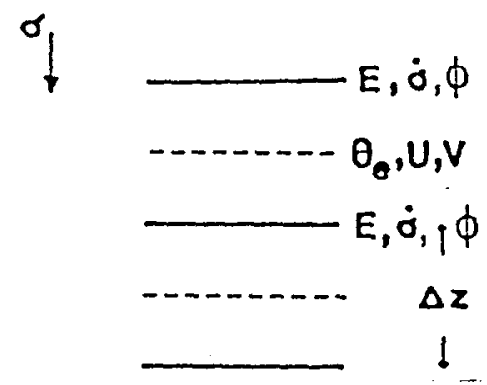

Fig. 2. Vertically staggered $\sigma$-coordinate.

horizontal grid interval ( $\Delta x$ and $\Delta y$ ) is $2 \mathrm{~km}$. The averaged vertical grid interval is about $80 \mathrm{~m}$, with higher resolution $(40 \mathrm{~m})$ near the water surface. Due to a staggered vertical grid arrangement (Figure 2), the lowest temperature grid point is placed at just $20 \mathrm{~m}$ above sea level. The domain consists of $71 \times 71 \times 37(=$ $186,517)$ grids.

Arakawa's (1972) C lattice was used for its efficiency in handling gravity waves. A forward-backward scheme (Sun, 1984) with a split-explicit time integration (Gadd, 1978) is used to avoid $2 \Delta t$ waves and to save computing time and memory. The time interval is $7.3 \mathrm{sec}$ for the adjustment stage of the integration for the terms involving inertia gravity waves. For the horizontal advection and diffusion terms, the integration time step is $21.6 \mathrm{sec}$. Details are presented in Sun and Hsu (1988).

\section{Initial Conditions and Boundary Conditions}

We simulated the convection which occurred over the East China Sea at $00 \mathrm{Z}$, February 16, 1975. At that time, the Air Mass Transformation Experiment (AMTEX) was in progress. Special acrological data collected at Keifu (see Figure 1 for location) is used to help set up the initial conditions and to compare with the numerical results. For initial conditions, it has been assumed that all of the atmospheric variables are uniformly distributed in horizontal planes (in $\sigma$-coordinates).

The initial basic model temperature, $T$, is assumed by

$$
T=T_{0}-\Gamma z
$$

where $T_{0}=279^{\circ} \mathrm{K}$ and $\Gamma=-\partial T / \partial z=4^{\circ} \mathrm{K} \mathrm{km}^{-1}$. This stratification is absolutely stable. The initial surface water temperature, $T_{\text {sea }}$, is set equal to $T_{0}$, in order to avoid numerical instability caused by the sharp discontinuity. During the first hour of integration, $T_{\text {sea }}$ is increased to the observed value of $289.4^{\circ} \mathrm{K}$, and then is kept constant with time. The initial specific humidity, $q$, is determined by:

$$
q=r q_{s}
$$


where $q_{s}$ is the saturated specific humidity and $r$, the relative humidity, is $40 \%$. Similar profiles of $T$ and $q$ have been used as the initial conditions by Asai and Nakamura (1978). The initial wind speed is $12 \mathrm{~m} / \mathrm{sec}$ in the positive $x$-direction at all levels. Surface pressure, $p_{s}$, is $1020 \mathrm{mb}$.

A cyclic condition is imposed at the lateral boundaries. A uniform geostrophic wind is prescribed. As for the upper boundary, a rigid lid is used.

Initially, the model atmosphere was too stable for MCC to develop. Hence, a one-dimensional model with the same set of governing equations was first integrated for $11 \mathrm{hr}$ (in physical time), in order to obtain a reasonable environment in which the depth of CBL was about $1.4 \mathrm{~km}$. Then the results were extended uniformly into a three-dimensional domain. The University of Purdue's 3D model is used with random temperature perturbations (magnitude of $0.3 \mathrm{~K}$ ) imposed in the CBL. After $2.4 \mathrm{hr}$, the MCCs were already quite intense. The results at time $t=13.4 \mathrm{hr}$, which includes 11 hours integration by the one-dimensional model and 2.4 hours by the three-dimensional model, will be presented in the next section.

\section{Results and Discussions}

It is convenient to decompose an instantaneous value of some meteorological parameter $\psi$, into three components:

$$
\psi=\langle\psi\rangle+\psi^{\prime \prime}+\psi^{\prime},
$$

where $\langle\psi\rangle$ is the basic state, or the horizontal average of $\psi$ over the entire domain, $\psi^{\prime \prime}$ is the resolvable component which is contributed mostly by $\mathrm{MCC}$, and $\psi^{\prime}$ is a subgrid-scale eddy component. As examples of the convention, $\overline{w^{\prime} \theta_{v}^{\prime}}$ is the ensemble-average eddy heat flux, and $\left\langle w^{\prime \prime} \theta_{v}^{\prime}\right\rangle$ denotes the horizontal average of vertical heat flux by MCC.

\subsection{Basic STATE OF THE CBL}

The solid line in Figure 3 depicts the vertical profile of simulated mean virtual potential temperature $\left\langle\theta_{v}\right\rangle$ at time $t=13.4 \mathrm{hr}$. Since we present the results at $t=$ $13.4 \mathrm{hr}$ only, the time will not be specified in the following discussions.

Also in Figure 3 are a dotted line showing the model initial $\theta_{v}$ profile, and a dashed line showing the $\theta_{v}$ sounding taken at 00Z, February 16, 1975 at Keifu. The initial model atmosphere was stable and cold near the surface $\left(\theta_{v}=5^{\circ} \mathrm{C}\right)$. Due to the transport of heat from the warm water, $\left\langle\theta_{v}\right\rangle$ increased in the lower level, and a CBL, characterized by uniformly distributed $\left\langle\theta_{v}\right\rangle$, developed. The stable air above the CBL remained undisturbed. The simulated $\left\langle\theta_{v}\right\rangle$ within the CBL is about 3 degrees warmer than the observed $\theta_{v}$. This is largely due to the fact that cold air advection is ignored in this model.

The model $\left\langle\boldsymbol{\theta}_{v}\right\rangle$ profile shows the multiple-layer structure of the CBL. They are: (1) superadiabatic surface layer in the lowest $20 \mathrm{~m}$ near the warm $\left(289.4^{\circ} \mathrm{K}\right)$ water; (2) subcloud layer, characterized by slightly decreasing $\left\langle\theta_{v}\right\rangle$ with height, located 


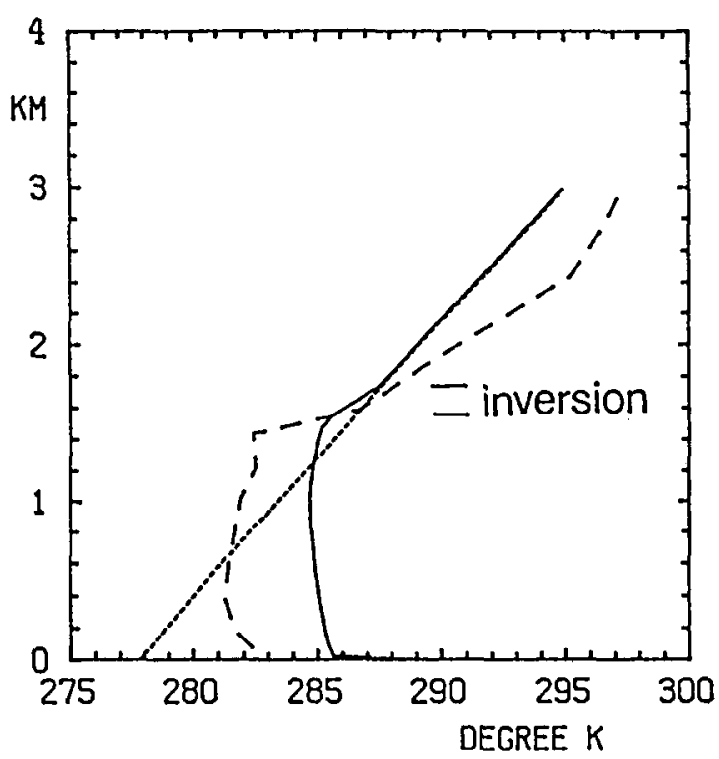

Fig. 3. Vertical profiles of virtual potential temperature. Model output $\left\langle\theta_{v}\right\rangle$ is the solid line, initial condition is the dotted line, and observed profile at Keifu, 00Z, February 16, 1975 is the dashed line.

over the top of the surface layer to about $1 \mathrm{~km}$ above sea level; (3) a layer filled with clouds and stable air between clouds, characterized by slightly increasing $\left\langle\theta_{v}\right\rangle$, with height due to condensation and evaporation; and (4) a very thin inversion layer capping the cloud layer. The CBL is defined as the combination of (1), (2), and (3).

Figure 4 shows the vertical profiles of specific humidity, $q$. At the upper CBL, $q$ decreases a little more sharply than that of the lower CBL. In the inversion layer, specific humidity, $q$, for both model output and the observed profiles, decreases rapidly with height, as the moisture transported from the water surface is mostly confined in the CBL. The simulated vertical profile of moisture content $\langle q\rangle$ agrees with the observed profile at Keifu, except that the true atmosphere was drier, mainly due to the large-scale dry advection over the region.

The distribution of mean specific liquid water content $\left\langle q_{l}\right\rangle$ is shown in Figure 5, which is consistent with the cloud layer observed at Keifu. The simulated mean wind in the CBL follows a modified Ekman spiral quite well (Figure 6). Crossisobaric flows, induced by the surface frictional stress, can be found within the CBL.

\subsection{Cloun configuration}

As mentioned earlier, a one-dimensional model is initially introduced to save computer time. Clouds start to form in the one-D model when the top of the CBL reaches $1.3 \mathrm{~km}$ (at the $10 \mathrm{th} \mathrm{hr}$ of integration). The three-D model was introduced 


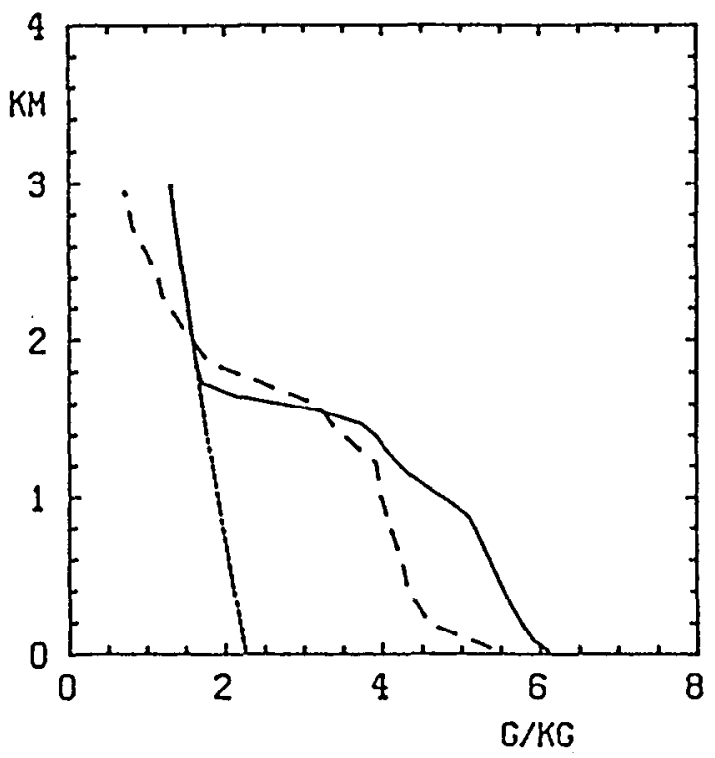

Fig. 4. Vertical profiles of specific humidity. The dotted, dashed, and solid lines are the initial $q$, observed $q$, and simulated $\langle q\rangle$, respectively.

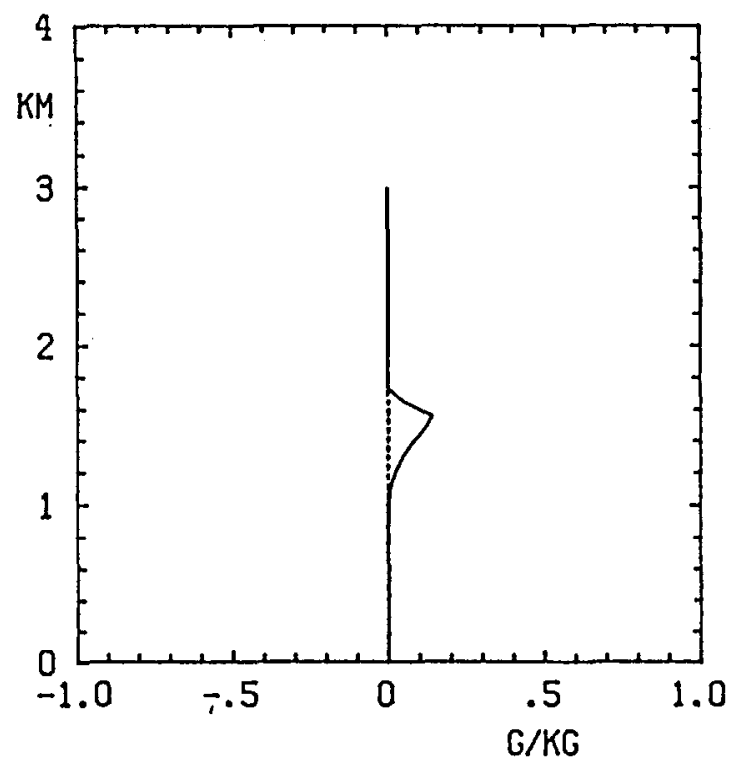

Fig. 5. Vertical profiles of specific liquid water content. $\left\langle q_{l}\right\rangle$ is the solid line and initial $q_{l}$ is the dotted line.

at the $11 \mathrm{th} \mathrm{hr}$ of integration. By the time the CBL grew to $1.6 \mathrm{~km}$, convection became very active. In the sinking branches of convection, warmer and drier air from the stable layer was entrained into the cloud layer and formed a clear region. This is called "layer cloud instability" by Moeng and Arakawa (1980). The 


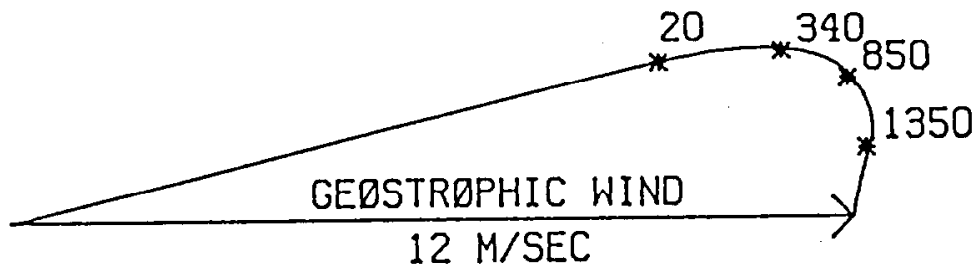

Fig. 6. Wind Hodograph $(\langle u\rangle,\langle v\rangle)$. Heights in meters.

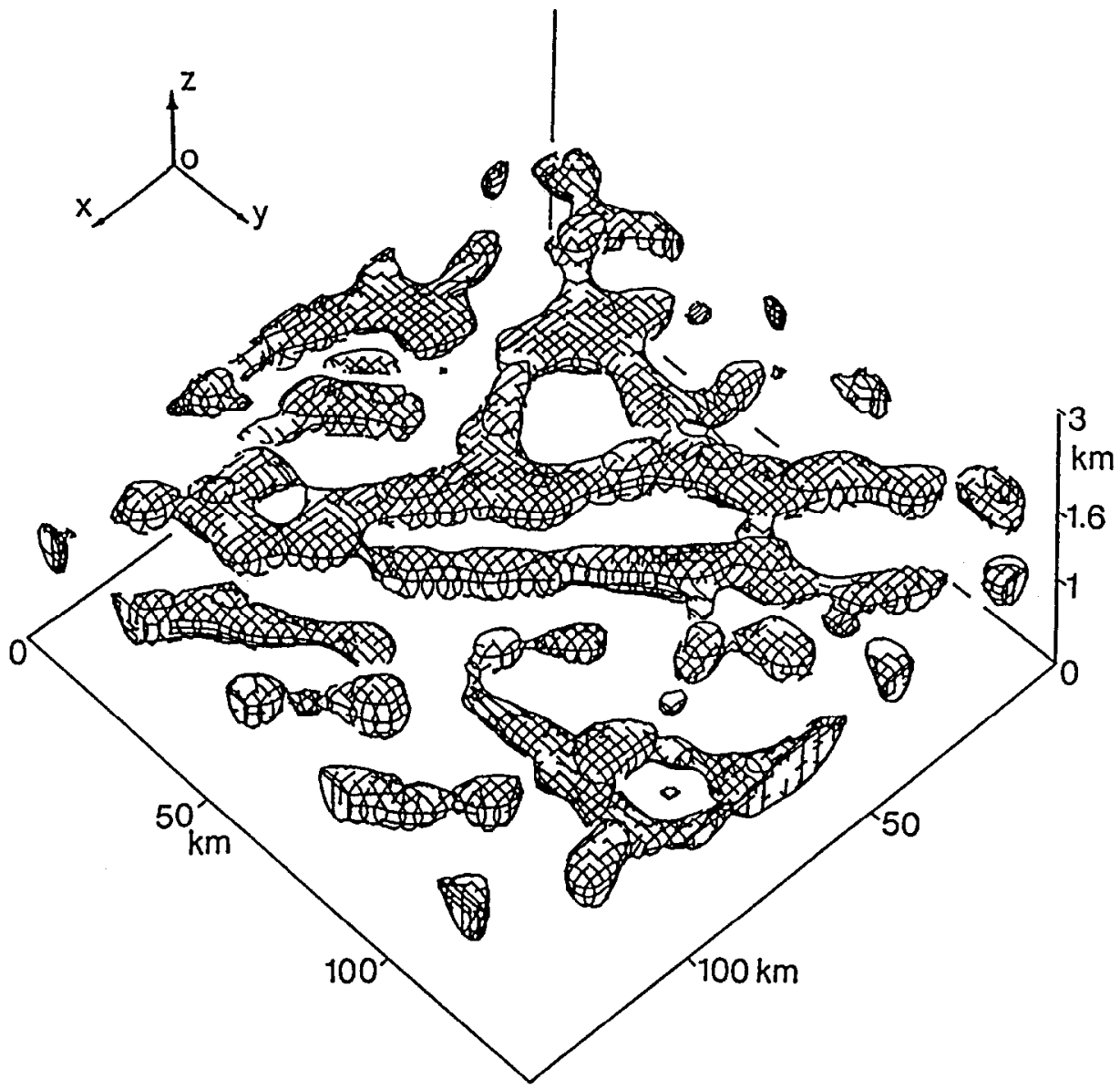

Fig. 7. Three-dimensional view of the liquid water distribution. Areas where $q_{l}>0.05 \mathrm{~g} / \mathrm{kg}$ are enclosed.

stratus cloud deck, covering the upper CBL at the start of the three-dimensional integration, subsequently broke into small patches of cumulus clouds.

Figure 7 presents the three-dimensional simulated clouds. A small inset diagram in the upper left corner shows the orientation of the coordinates. The origin of 
the domain is indicated by " $\mathrm{O}$ " in the inset diagram. In the background, a large diamond (beneath the clouds) depicts the horizontal domain of the numerical model at sea level. Below $1 \mathrm{~km}$ is the subcloud layer. All of the clouds are confined to the upper portion of the CBL (1 to $1.6 \mathrm{~km}$ ). The undisturbed stable layer extends from $1.6 \mathrm{~km}$ to the top of the domain $(3 \mathrm{~km})$.

The simulated clouds appear to be mixed cells and rolls. There are three complete "open" cells and some broken MCCs. However, the imposed artificial periodic lateral boundary condition distorts MCCs to roll structure. This is evident because these rolls are oriented in a diagonal direction.

One possible explanation for cells appearing open instead of closed in the center is offered by Moeng and Arakawa (1980): heating from below by a warm sea surface tends to narrow the zones of upward motion so that open cells form. As we shall see in the next subsection, simulated upward motion is indeed confined to small areas.

A statistical study by Agee (1976) shows that the sizes of the convective cells are a function of the CBL depth and the total flux of sensible heat and latent heat from the water surface. Figure 3 illustrates that the simulated depth of the CBL is only slightly higher than the observed depth at Keifu. The simulated total heat flux from the surface is $425 \mathrm{~W} / \mathrm{m}^{2}$, including $110 \mathrm{~W} / \mathrm{m}^{2}$ contributed from sensible heat and $325 \mathrm{~W} / \mathrm{m}^{2}$ from latent heat. This total amount also matches the observed value of $400 \mathrm{~W} / \mathrm{m}^{2}$ (at Keifu, 00Z, February 16, 1975) reported by Sheu and Agee (1977). Since both conditions are well simulated, we can compare the sizes of the simulated MCC and the sizes of the MCC shown in the satellite picture (Figure 1).

The averaged cell size (diameter) over Keifu at 0235Z, February 16, 1975 in Figure 1 is $20 \mathrm{~km}$, while the averaged simulated cell size is $23 \mathrm{~km}$. The aspect ratio for our simulated MCC, $14.3(23 \mathrm{~km}$ divided by $1.6 \mathrm{~km})$, agrees well with the observed aspect ratio of $13.3(20 \mathrm{~km}$ divided by 1.5$)$. One might suspect that the large ratio of horizontal to vertical grid spacing forces flat cells, but we believe that these cells are physically meaningful due to the high spatial resolution used in the model.

While the horizontally-averaged specific liquid water content, $\left\langle q_{l}\right\rangle$, at the top of the cloud layer is only slightly larger than $0.1 \mathrm{~g} / \mathrm{kg}$ (see Figure 5), the $q_{l}$ in the center of a cumulus can be as large as $0.8 \mathrm{~g} / \mathrm{kg}$. Figure 8 shows a vertical profile of $q_{l}$ where the specific liquid water content is the largest in the CBL. This vertical profile of $q_{I}$ (marked by labels of 1's) is very close to the profile simulated in Deardorff's (1980) model. Another line (marked by labels of 2's) in Figure 8, is the vertical profile of $q_{l}$, where specific humidity $q$ is the smallest in the CBL. There is no cloud ( $q_{l}=0$ at all heights) at that particular location. In order to analyze the structures of the convective cells, we shall compare the vertical profiles of many atmospheric parameters for these two locations. For convenience, we shall categorize them as column 1 (in a cloudy region) and column 2 (in a cloud- 


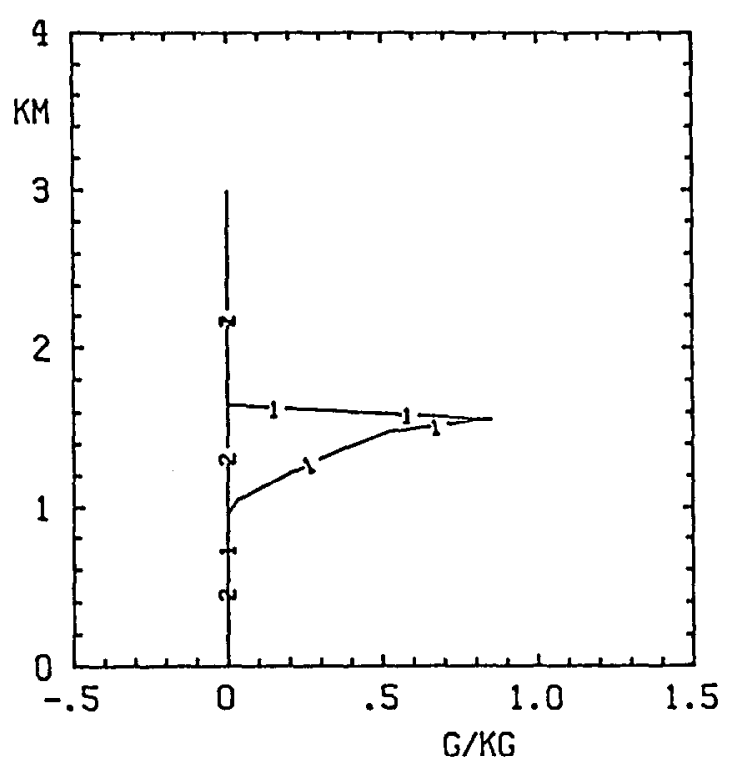

Fig. 8. Vertical profiles of specific liquid water content, $q_{l}$, for column 1 in a cloudy region and column 2 in a cloud-free region.

free region). The $x-z$ vertical cross-section of the distribution of $q_{l}$ at $y=70 \mathrm{~km}$ is shown in Figure 9. One can clearly see the five cumulus clouds along the $x$ direction.

\subsection{Kinematic aspects of the simulated MCC}

A composite view of the MCC component of wind speed, $u^{\prime \prime}(=u-\langle u\rangle)$, and vertical motion, $w$, best demonstrates the convective motion (Figure 10). The scales of the velocity vectors are shown at the upper right of the figure. Note that the vertical motion has been exaggerated by a factor of 3 to demonstrate the convective motion. No significant vertical motion is found in the stable layer above. The upward motion is more intense than the downward motion. At height $z=1.1 \mathrm{~km}$, where the vertical motion is strongest, the area covered by the upward motion is only about one half of the area covered by the downward motion.

The maximum simulated upward motion in column 1 is nearly $0.25 \mathrm{~m} / \mathrm{sec}$, while the maximum downward motion in column 2 is only about $0.15 \mathrm{~m} / \mathrm{sec}$ (Figure 11). Another interesting feature is that the maximum upward motion is achieved at the upper CBL while the maximum downward motion is located near the middle of the CBL. As an air parcel moves upward in the cloudy region from the water surface, it experiences acceleration due to the unstable environmental condition. When the parcel moves up to the cloud layer, latent heat is added to the air parcel. The upward motion is stopped when the parcel penetrates the inversion layer. As for the downward motion in a cloud-free region, there is no latent energy available. It will be shown later in Section 4.5 that the upper CBL in a cloud-free 


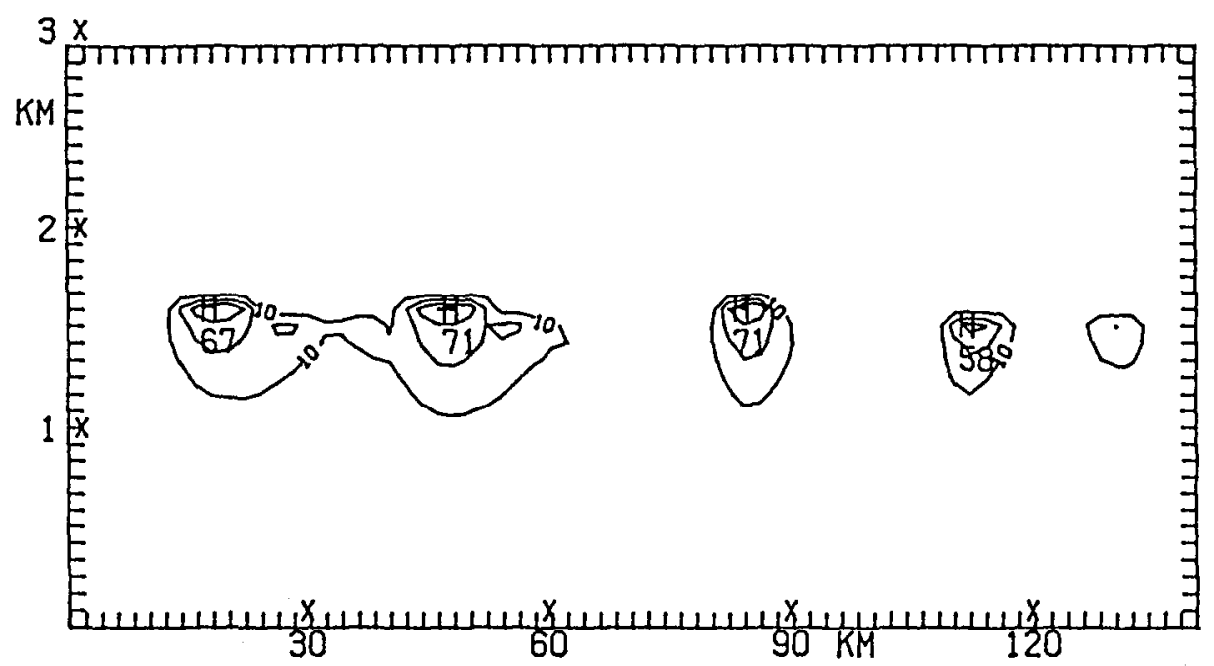

Fig. 9. Vertical cross-section of specific liquid water content, $q_{l}$, at $y=70 \mathrm{~km}$, with contour interval of $0.2 \mathrm{~g} / \mathrm{kg}$; labels scaled by $10^{5}$

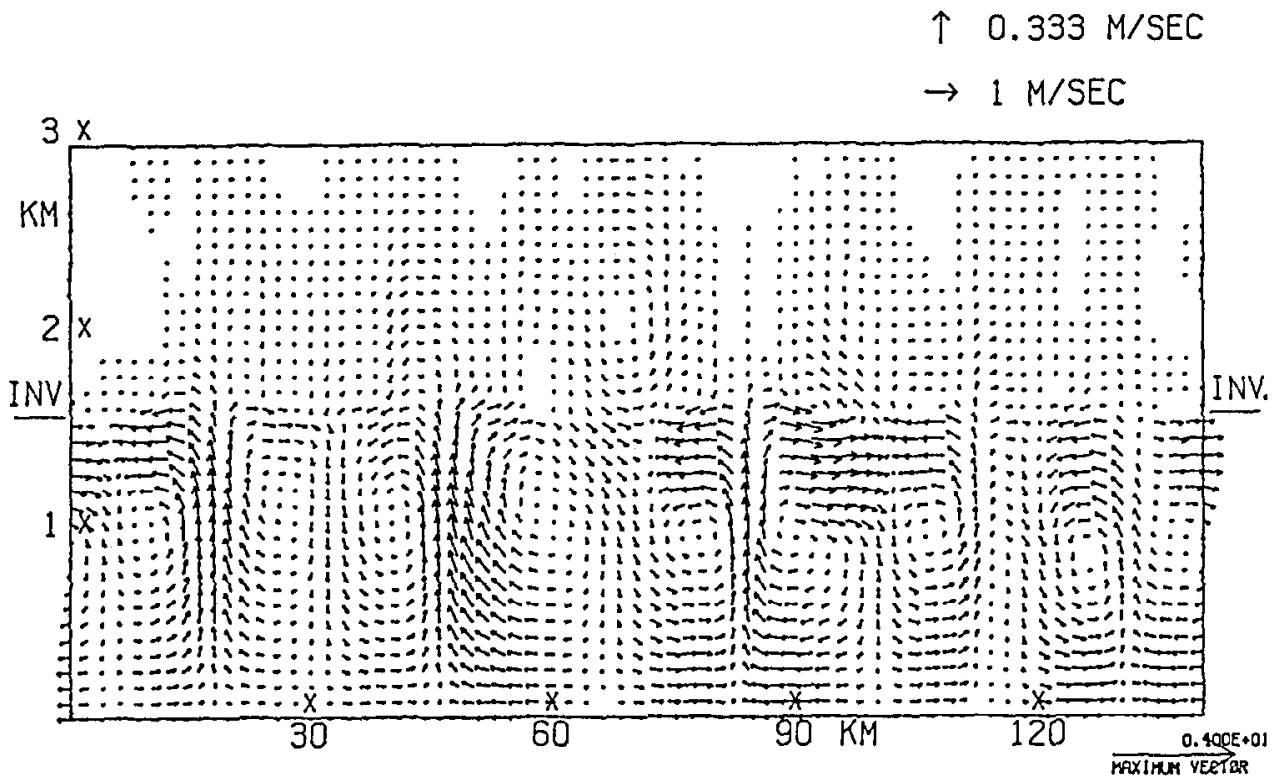

Fig. 10. Vertical cross-section of velocity vector, $\left(u^{\prime \prime}, w\right)$, at $y=70 \mathrm{~km}$. Scales for the vector lengths are shown in the upper right corner.

region is actually stable. The downward motion is somewhat inhibited due to the adiabatic warming effect. However, the downward motion can still be maintained, partially due to the returning air mass from the strong upward motion in cloudy regions.

The skewness of the vertical velocity is also reported by other numerical studies 


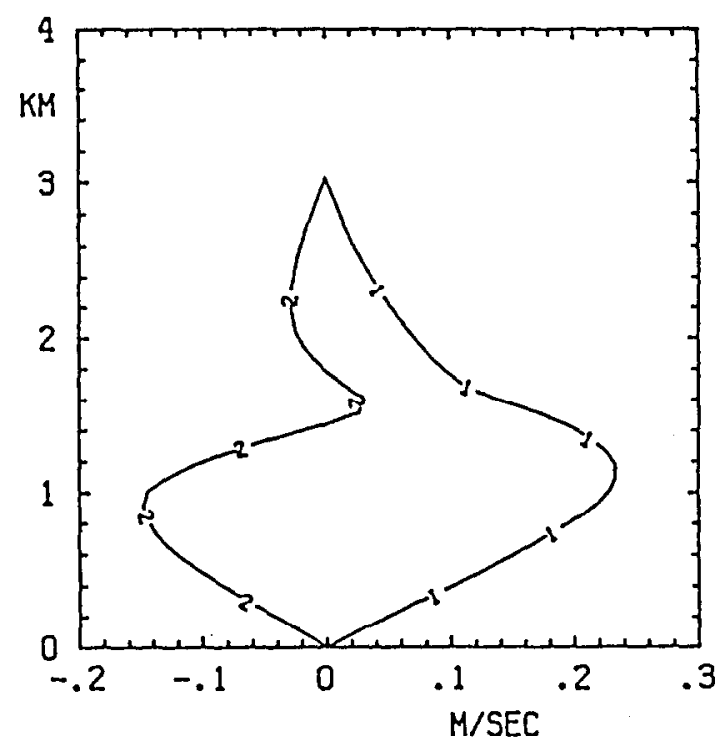

Fig. 11. Same as Figure 8, except for vertical motion, $w$.

and observations. As mentioned earlier, strong and narrow upward motion possibly induces open cells.

\subsection{TeMPERATURE AND MOISTURE DistribUtion}

Figure 12 shows the vertical profiles of virtual potential temperature, $\theta_{v}$, for column 1 (in a cloudy region) and column 2 (in a cloud-free region). For column $1, \theta_{v}$ is higher than for column 2 in the $\mathrm{CBL}$ because column 1 represents the location of a hot plume. In the cloud layer for column $1, \theta_{\nu}$ increases with height according to the moist adiabatic lapse rate. Such a lapse rate is notably absent for the vertical profilc of $\theta_{v}$ for column 2 (cloud-frce area). The temperature difference between the cell center and wall of the $\mathrm{MCC}$ can be as large as $0.8^{\circ} \mathrm{K}$ due to the release of latent heat and convection. At the cloud base, the difference is much smaller $\left(0.25^{\circ} \mathrm{K}\right)$. This value is comparable with an average difference $\left(0.26^{\circ} \mathrm{K}\right.$, just under a cloud layer) found in Rothermel and Agee's (1980) aircraft investigation of a closed cell during AMTEX 75 (no such data are available for an open cell).

The inversion layer, defined as the layer in which $\theta_{v}$ increases rapidly with height, is higher for the ascending branch of the convective cell (at $1600 \mathrm{~m}$ for column 1) than the inversion layer for column 2 (at $z=1440 \mathrm{~m}$, Figure 12). Figure 13 shows the undulation of the inversion height with vertical motion. Tightly packed contour lines of $\theta_{\nu}$ signify the location of the inversion layer. This undulation of the inversion height has also been reported by Kuo and Sun (1976) and others. The area with greater inversion height matches the location of the upward motion shown in Figure 10. 


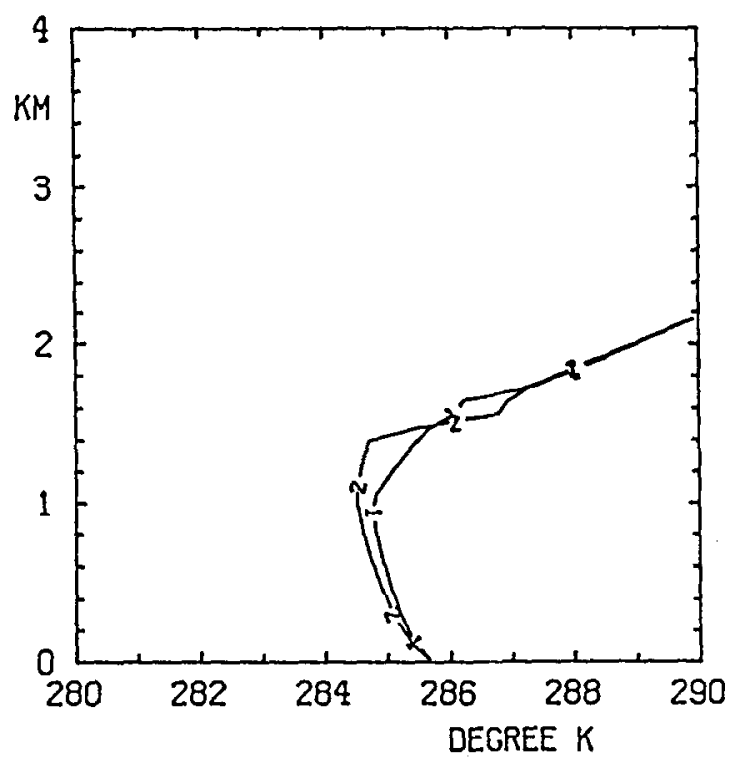

Fig. 12. Same as Figure 8, except for virtual potential temperature, $\theta_{v}$.

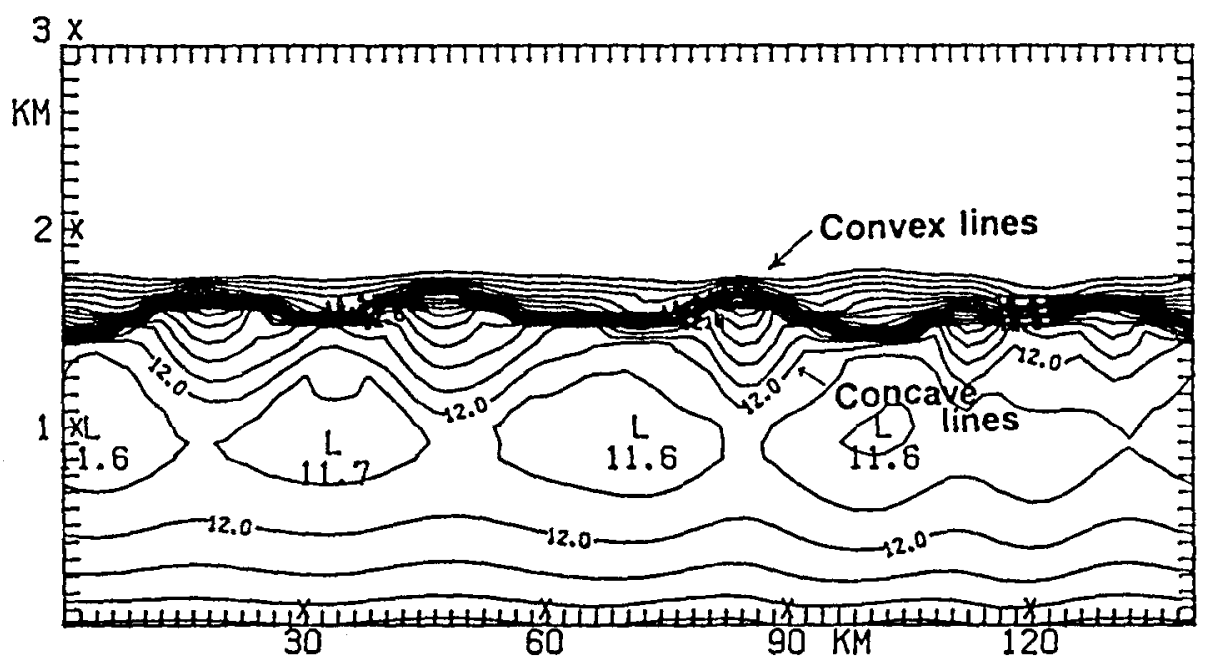

Fig. 13. Vertical cross-section of virtual potential temperature at $y=70 \mathrm{~km}$, with contour interval of $0.2^{\circ} \mathrm{C}$, labels in ${ }^{\circ} \mathrm{C}$.

The curved contour lines immediately above the inversion layer are the results of the penetrative upward motions. An upward-moving air parcel originating from the CBL will be stopped by the inversion with temperature even higher than its own. Directly below the inversion in the cloudy region, contour lines are concave downward in the cloud layer, as the parcel is warmer than the surrounding air. These areas (with concave-downward contour lines) match the areas with clouds 


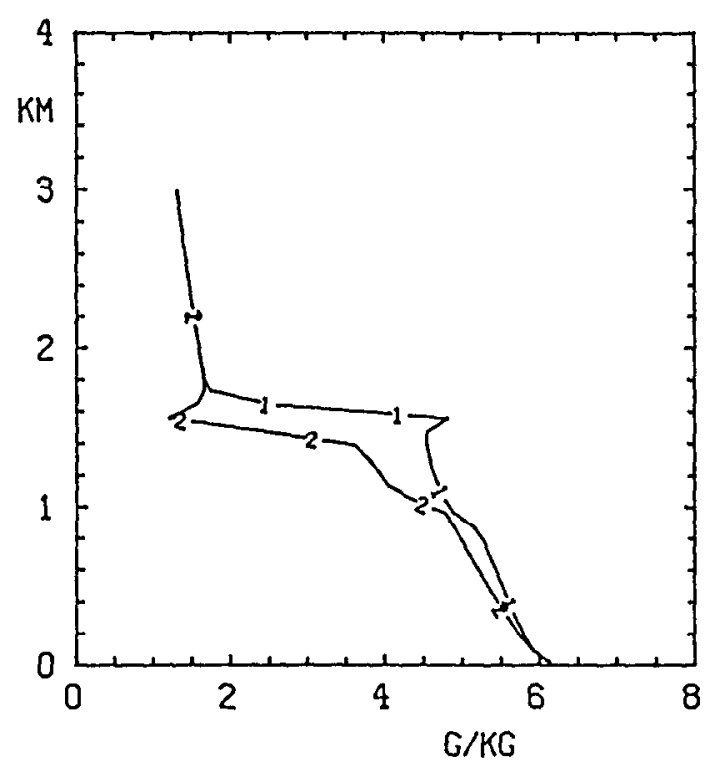

Fig. 14. Same as Figure 8, except for specific humidity, $q$.

shown in Figure 9. They are also characterized by the strongest $\theta_{v}$ gradients inside the entire CBL, except in the surface layer. This is due to the moist-adiabatic temperature lapse rate in the region, whereas $\theta_{v}$ is uniformly mixed with the dryadiabatic lapse rate in other parts of the CBL. The variation of $\theta_{v}$ in the lower $\mathrm{CBL}$ is much smaller.

Figure 14 depicts the vertical profiles of specific humidity, $q$, for the cloudy and cloud-free regions. Specific humidity is $0.6 \mathrm{~g} / \mathrm{kg}$ larger for the ascending branch of the convective cell than for the cloud-free region in the upper CBL. Rothermel and Agee (1980) showed that the difference is $0.7 \mathrm{~g} / \mathrm{kg}$ near the cloud base during AMTEX 75, which is close to the simulated results.

Figure 15 contains the vertical cross-section of specific humidity, $q$, at $y=70 \mathrm{~km}$. Contour lines with values less than $3 \mathrm{~g} / \mathrm{kg}$ are not drawn. The inversion layer is characterized by a sharp decrease of $q$ with height. Moist regions are associated with warmer temperature and ascending motion in the CBL. In the lower CBL, the ridges and troughs of the contour lines tilt slightly toward the right with height. This indicates that the convective cells are not perfectly stacked in the vertical direction, since the mean wind (from left to right) is weaker near the water surface (see Figure 6).

\subsection{TIIRRIIIFNT FIIIXFS}

The linear decrease of $\left\langle\overline{w^{\prime} \theta_{v}^{\prime}}\right\rangle$ (horizontally-averaged turbulent flux, solid line in Figure 16) with height in the lower CBL ensures a uniform increase of mean temperature with time in the subcloud layer. The second maximum of heat located in the vicinity of the cloud layer, is related to the appearance of clouds. As the 


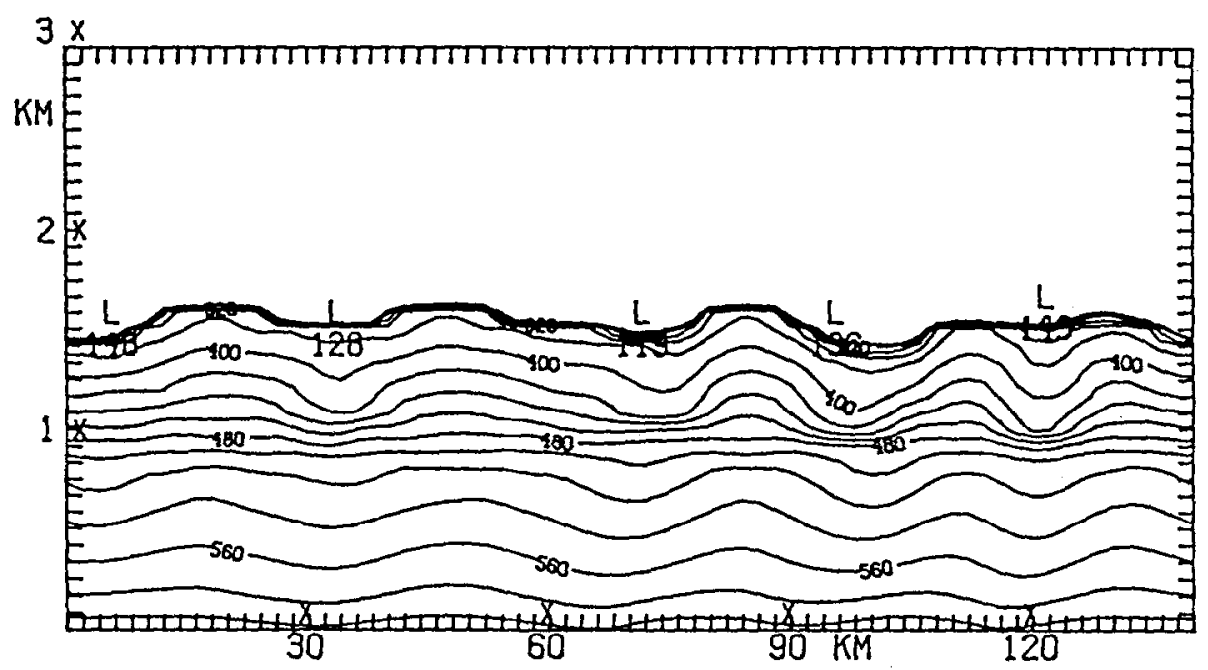

Fig. 15. Vertical cross-section of specific humidity, $q$, at $y=70 \mathrm{~km}$, with contour interval of $0.2 \mathrm{~g} / \mathrm{kg}$, labels scaled by $10^{5}$.

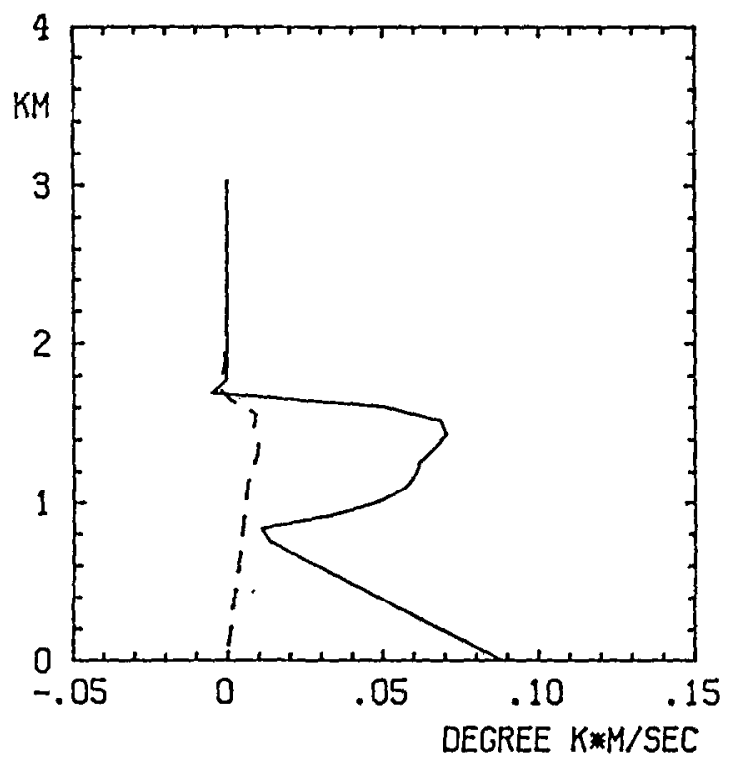

Fig. 16. Vertical profiles of buoyancy flux. $\left\langle\overline{w^{\prime} \theta_{v}^{\prime}}\right\rangle$ is the solid line, and $\left\langle\overline{w^{\prime \prime} \theta_{v}^{\prime \prime}}\right\rangle$ is the dashed line.

CBL grows thicker with time, the capping inversion gradually shifts upward, and the cloud layer also moves toward higher levels.

Figure 17 shows the vertical profile of horizontally-averaged liquid water flux $\left\langle\overline{w^{\prime} q_{l}^{\prime}}\right\rangle$ by turbulent eddies. The decrease of $\left\langle\overline{w^{\prime} q_{l}^{\prime}}\right\rangle$ in the lower portion of the cloud layer and the convergence of $\left\langle\overline{w^{\prime} q_{l}^{\prime}}\right\rangle$ in the upper portion of the cloud layer suggest that the ascension of the cloud layer (in the "horizontal average" sense) 


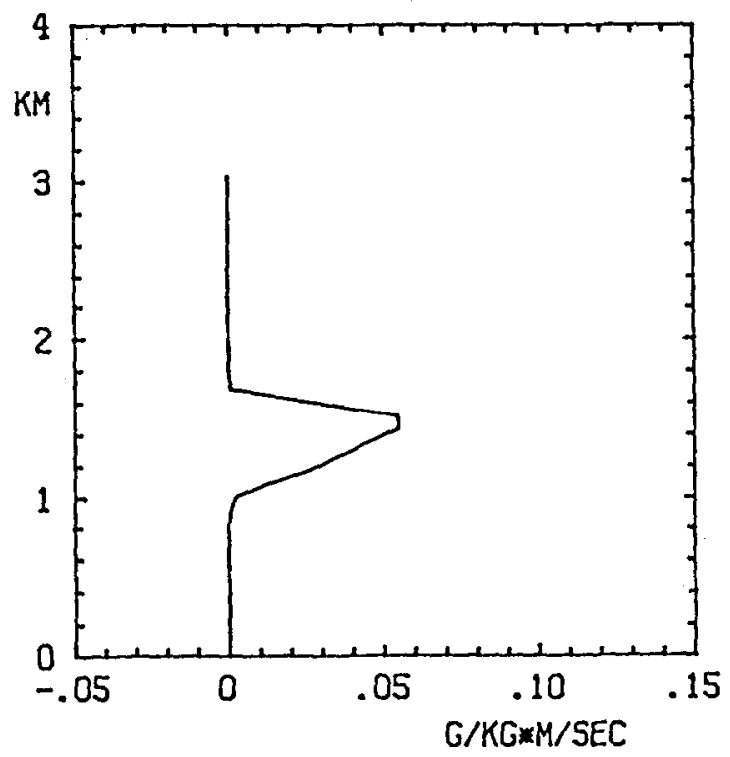

Fig. 17. Vertical profile of turbulent liquid water flux $\left\langle\overline{w^{\prime} q_{l}^{\prime}}\right\rangle$.

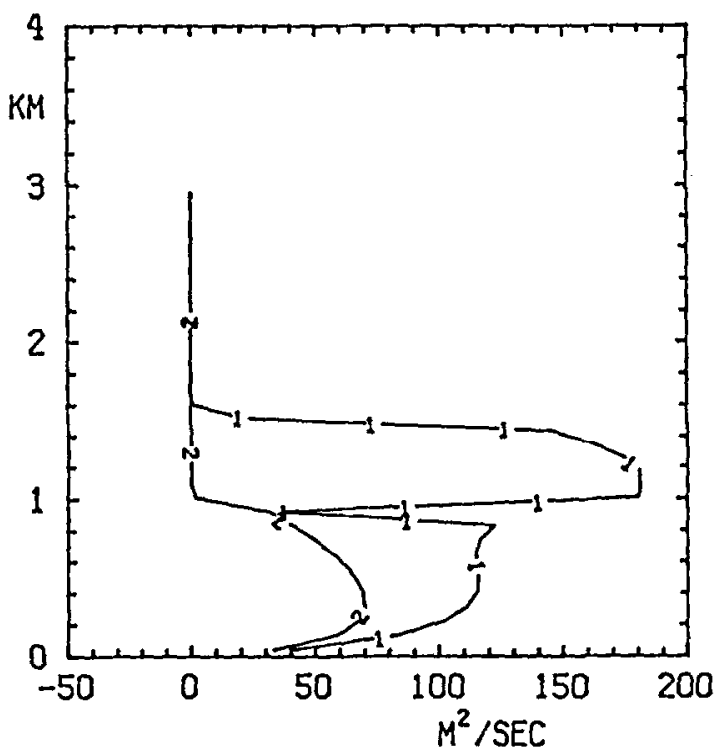

Fig. 18. Same as Figure 8, except for the eddy coefficient for heat, $K_{h}$.

is partly due to eddy transport of liquid water, and partly due to condensation near the cloud top.

The vertical profiles of eddy heat coefficient, $K_{h}$, for columns 1 and 2 are depicted in Figure 18. A large value of $K_{h}$ signifies an unstable atmospheric condition and active turbulence producing well-mixed temperature and moisture 
fields. The value of $K_{h}$ is very large for column 1 in the cloud layer as the air becomes extremely turbulent, forced by the release of latent heat. In the absence of this mechanism in column $2, K_{h}$ is almost zero above $1 \mathrm{~km}$. This implies a stable condition due to entrainments and adiabatic warming. Thus, the turbulent fluxes are distributed quite unevenly inside the CBL. The upward heat and moisture transport from the ocean surface are mainly achieved through the upward branches of the MCCs (particularly true in the upper CBL).

We can conclude that the MCCs play an important role in creating a favorable environmental condition for clouds to develop in the upward branch of the convection. Eddy fluxes are greatly enhanced once the clouds appear in these areas. It is possible that the overall heat and moisture fluxes may be smaller without these MCCs. One other thing worth noting is that the MCCs, however, transport little heat $\left(\left\langle\overline{w^{\prime \prime} \theta_{v}^{\prime \prime}}\right\rangle\right.$, dashed line in Figure 16) and moisture themselves compared to that of the turbulent eddies.

\section{Summary}

We have simulated MCC observed over the East China Sea during cold air outbreaks in the winter season with a high-resolution, three-dimensional numerical model.

The simulated CBL is capped by an inversion with strong temperature and moisture gradients. The temperature in the CBL is slightly colder than the observed atmospheric temperature, but the total heat flux in the model is comparable with that observed.

With strong upward motion concentrated in narrow regions surrounding broad cloud-free areas, the simulated convective cells are "open" in the center. The averaged aspect ratio of the cells is 14 , which agrees with the observed aspect ratios of convective cells over the East China Sea. Even though the heat fluxes generated by MCCs themselves are very small compared with the unresolvable eddy heat flux, MCCs create favorable environmental conditions for clouds to develop in the upward branches of convection. Thus, MCCs appear to be quite important in maintaining large eddy heat and moisture fluxes from the ocean surface.

\section{Acknowledgements}

The authors would like to extend their thanks to Professors E. Agee, P. Smith, and D. Vincent for their invaluable comments. This work is sponsored by the National Science Foundation of the United States under grants ATM-821965 and ATM-8313418. Part of this research was supported by The National Science Council of the Republic of China (Taiwan) under project NSC76-0202-M002-18. 


\section{References}

Agee, E. M.: 1976, 'Observational Evidence of Cell Flatness as a Function of Convective Depth and Eddy Anisotropy', J. Meteorol. Soc. Japan, 54, 68-71.

Agee, E. M., Chen, T. S., and Dowell, K. E.: 1973, 'A Review of Mesoscale Cellular Convection', Bull. Amer. Meteorol. Soc. 54, 1004-1012.

Arakawa, A.: 1972, 'Design of the UCLA General Circulation Model', Numerical Simulation of Weather and Climate, Tech. Rept. 7, Dept. of Meteorology, Univ. of California, Los Angeles.

Asai, T. and Nakamura, K.: 1978, 'A Numerical Experiment of Airmass Transformation Processes over Warmer Sea. Part 1: Development of a Convectively Mixed Layer', J. Meteorol. Soc. Japan, 56, 424-434.

Benard, H.: 1901, 'Les Tourbillons Cellulaires dans une Nappe Liquide Transportant de la Chaleur par Convection en Regime Permanent', Ann. Chim. Phys. 23, 62-144.

Deardorff, J. W.: 1974a, 'Three-Dimensional Numerical Study of the Height and Mean Structure of a Heated Planetary Boundary Layer', Boundary-Layer Meteorol. 7, 81-106.

Deardorff, J. W.: 1974b, 'Three-Dimensional Numerical Study of Turbulence in an Entraining Mixed Layer', Boundary-Layer Meteorol. 7, 199-226.

Deardorff, J. W.: 1976, 'Usefulness of Liquid-Water Potential Temperature in a Shallow-Cloud Model', J. Appl. Meteorol. 15, 98-102.

Deardorff, J. W.: 1980, Stratocumulus-Capped Mixed Layers Derived from a Three-Dimensional Model', Boundary-Layer Meteorol. 18, 495-527.

Fiedler, B. H.: 1984, 'The Mesoscale Stability of Entrainment into Cloud-Topped Mixed Layer', $J$. Atmos. Sci, 41, 92-101.

Gadd, A. J.: 1978, 'A Split Explicit Integration Scheme for Numerical Weather Prediction', Quart. J. Roy. Meteorol. Soc. 104, 569-582.

Krishnamurti, R.: 1975a, 'On Cellular Cloud Patterns. Part 1: Mathematical Model', J. Atmos. Sci. 32, 1353-1363.

Krishnamurti, R.: 1975b, 'On Cellular Cloud Patterns. Part 3: Applicability of the Mathematical and Laboratory Models', J. Atmos. Sci. 32, 1373-1383.

Kuo, H. L. and Sun, W. Y.: 1976, 'Convection in the Lower Atmosphere and Its Effects', J. Atmos. Sci. 33, 21-40.

Miura, Y.: 1986, 'Aspect Ratios of Longitudinal Rolls and Convection Cells Observed During Cold Air Outbreaks', J. Atmos. Sci. 43, 26-39.

Moeng, C.-H. and Arakawa, A.: 1980, 'A Numerical Study of a Marine Subtropical Stratus Cloud Layer and its Stability', J. Atmos. Sci. 37, 2661-2676.

Ninomiya, K.: 1975, 'Large-Scale Aspects of Air-Mass Transformation Over the East China Sea During AMTEX '74', J. Meteoral. Soc. Japan, 53, 285-303.

Priestley, C. H. B.: 1962, 'The Width-Height Ratio of Large Convection Cells', Tellus 14, 123-124.

Rayleigh, O. M.: 1916, 'On Convection Currents in a Horizontal Layer of Fluid when the Higher Temperature is on the Under Side', Phil. Mag. 32, 529-546.

Rothermel, J. and Agee, E. M.: 1980, 'Aircraft Investigation of Mesoscale Cellular Convection During AMTEX 75', J. Atmos. Sci. 37, 1027-1040.

Sheu, P. J. and Agee, E. M.: 1977, 'Kinematic Analysis and Air-Sea Heat Flux Associated with Mesoscale Cellular Convection During AMTEX 75, J. Atmos. Sci. 34, 793-801.

Sommeria, G. and LeMone, M. A.: 1978, 'Direct Testing of a Three-Dimensional Model of the Planetary Boundary Layer Against Experimental Data', J. Atmos. Sci. 35, 25-39.

Sun, W. Y.: 1976, 'Linear Stability of Penetrative Convection', J. Atmos. Sci. 33, 1911-1920.

Sun, W. Y.: 1984, 'Numerical Analysis for Hydrostatic and Nonhydrostatic Equations of InertialInternal Gravity Waves', Mon. Wea. Rev. 112, 259-268.

Sun, W. Y. and Wu, C. C.: 1987, 'Numerical Study of the Diurnal Variation Near and at the Ground Surface' (Preprint of the 18th Conference on Agricultural and Forest Meteorology and 8th Conference on Biometeorology and Aerobiology, W. Lafayette, IN., Sept. 15-18).

Sun, W. Y. and Hsu, W.-R.: 1988, 'Numerical Study of a Cold Air Outbreak Over the Ocean', $J$. Atmos. Sci. 45, 1205-1227.

Sykes, R. I., Lewellen, W. S., and Henn, D. S.: 1988, 'A Numerical Study of the Development of Cloud-Street Spacing', J. Atmos. Sci. 45, 2556-2569. 